\title{
Pensamiento crítico y el rendimiento académico en la escuela de educación - universidad nacional Santiago Antúnez de Mayolo; 2019
}

\section{Lucero del Pilar Rodríguez Zúñiga}

lucerod@hotmail.com

Universidad Nacional Mayor de San Marcos

Perú - Huaraz

\section{RESUMEN}

El presente artículo tuvo como objetivo determinar como el Pensamiento Crítico influye en el Rendimiento Académico en la Escuela de Educación, Universidad Nacional Santiago Antúnez De Mayolo - 2019. Se consideró a 57 docentes nombrados de dicha Universidad. De los resultados se desprende que el análisis de información influye favorablemente en el Rendimiento Académico en la Escuela de Educación, además se comprobó que la inferencia de implicancias influye positiva y significativamente en el Rendimiento Académico en la Escuela de Educación. En líneas generales, los resultados demostraron que el pensamiento crítico influye de manera directa y significativa en el Rendimiento Académico en la Escuela de Educación, esto se aprecia a un coeficiente de correlación Rho de Spearman de 0,768, evidenciando una correspondencia alta entre el Pensamiento Crítico y el Rendimiento Académico.

Palabras clave: pensamiento crítico; rendimiento académico y conocimientos. 


\title{
Critical thinking and academic performance in the school of education - universidad nacional Santiago Antúnez de Mayolo; 2019
}

\begin{abstract}
The objective of this article was to determine how Critical Thinking influences Academic Performance in the School of Education, National University Santiago Antúnez De Mayolo - 2019. 57 appointed teachers from said University were considered. The results show that the analysis of information favorably influences the Academic Performance in the School of Education, it was also found that the implication inference positively and significantly influences the Academic Performance in the School of Education. In general terms, the results showed that critical thinking directly and significantly influences Academic Performance in the School of Education, this is seen at a Spearman Rho correlation coefficient of 0.768 , showing a high correspondence between Critical Thinking and Academic Performance.
\end{abstract}

Keywords: critical thinking, academic performance and knowledge

Artículo recibido: 30 noviembre. 2021 Aceptado para publicación: 29 diciembre 2021

Correspondencia: lucerod@ hotmail.com Conflictos de Interés: Ninguna que declarar 


\section{INTRODUCCIÓN}

El pensamiento crítico se define como el proceso consciente y deliberado que se utiliza para interpretar o evaluar información y experiencias con un conjunto de actitudes y habilidades que guíen las creencias fundamentales y las acciones (Mertes, 1991). En Latinoamérica y en el Perú las Escuelas de Educación, en las que se forman a los formadores por excelencia y los "otros" no han recibido apoyo decidido del sector público, por lo que su contribución a la resolución de la problemática de la sociedad ha sido mínima, y las áreas prioritarias del conocimiento no están siendo debidamente atendidas, por lo que sigue siendo un reto propiciar cambios en el aprendizaje del alumnado de las diferentes escuelas existentes.

Durante el desarrollo de las sesiones de clase con los estudiantes la Escuela Académico Profesional de Lenguas, Literatura de la Escuela de Educación de la Universidad Nacional Santiago Antúnez de Mayolo en Huaraz, se ha observado algunas de las limitaciones en el uso del pensamiento crítico descritas en el párrafo anterior. Por ejemplo, son pocos los alumnos que plantean preguntas en clase, la mayoría recibe pasivamente la información que imparte el docente. Cuando se les plantea actividades que demandan análisis y reflexión sobre la información que han leído o escuchado, sus participaciones son breves y con poco fundamento.

Asimismo, se ha constatado que el rendimiento académico de los estudiantes de la facultad de Educación no es bueno, pues la mayoría obtiene calificaciones entre 11 y 14. De continuar esta situación descrita, no se va a contar con buenos educadores, formadores de profesionales y educadores de quienes van a construir el futuro de nuestro país.

Por lo tanto, este escrito resulta ser de sumo interés dado que los resultados servirán para recomendar se implemente la difusión de este tipo de pensamiento en la Escuela en estudio, lo cual servirá para contar con estudiantes autónomos, que busquen el conocimiento de manera guiada por el criterio y la duda, y que busque en el conocimiento la alternativa de solución de los diversos problemas que aquejan a nuestra sociedad.

\section{MATERIALES Y MÉTODOS}

\subsection{Estudios aproximados a las variables}

Huaranca (2018) en su estudio pudo determinar que existe relación significativa entre el uso del pensamiento crítico y el rendimiento académico en los estudiantes de la escuela profesional de contabilidad de la universidad católica los ángeles de Chimbote filial 
Juliaca con un coeficiente de correlación de 0,643. Es decir, pensamiento crítico influye de manera directa y moderada en el rendimiento académico; además el coeficiente de determinación, indica que el rendimiento académico se ve influenciada en un 41,29\% por el pensamiento crítico.

Así mismo se encontró el estudio de Alquichire y Arrieta (2018) cuyo resultado demuestra que no existe relación entre las habilidades de pensamiento crítico y el rendimiento académico; esto, mediante el estudio de una muestra de estudiantes de primer semestre de la Licenciatura en Biología y Química de la Universidad del Atlántico, con un resultado de la correlación de 0,004<0,05 y un nivel de confianza del 95\%.

En otro contexto, se recogió a Del Bustos (2017) quien por medio de su estudio pudo establecer que el adecuado desarrollo del pensamiento crítico se relaciona positivamente con el rendimiento académico en el área de Formación Ciudadana y Cívica, esta relación se verificó al encontrarse correlaciones estadísticamente significativas entre el rendimiento académico de estudiantes de cuarto y quinto grado de secundaria, por lo que pudo concluir que, los estudiantes con mayores puntajes en el rendimiento académico obtienen mejores resultados en el test; en consecuencia, el desarrollo del pensamiento crítico se relaciona positivamente con el rendimiento académico del área de Formación Ciudadana y Cívica.

En esa línea, se consideró la investigación de Alquichire y Arrieta (2018) donde se observa que entre el análisis y el rendimiento presentaron un coeficiente de correlación con una significancia de 0.0012 , por lo que no una correlación entre el análisis y el rendimiento académico.

\subsection{Aproximaciones al concepto de las variables}

Burton, Kimball y Wing (1965) afirman que el pensamiento crítico es un proceso que permite interpretar y resolver problemas, partiendo de los conocimientos que se poseen y de aquellos que se van adquiriendo. El término pensamiento significa la búsqueda crítica y reflexiva de conclusiones válidas que resuelvan problemas y dudas, permitiendo elegir entre afirmaciones antagónicas donde aparece constantemente en la vida. Esta idea permite afirmar que, en la medida que el pensamiento se mejora se va dando lugar a la transformación del mismo en pensamiento crítico. Mertes (1991) afirma que el pensamiento crítico es un proceso consciente y deliberado que se utiliza para interpretar 
o evaluar información y experiencias con un conjunto de actitudes y habilidades que guíen las creencias fundamentales y las acciones.

Por su parte Lipman (1991) refiere que los individuos utilizan, en un contexto dado, procesos críticos de pensamiento que les permiten distinguir la información más relevante de la menos relevante en relación con sus metas. Así, el pensamiento crítico es una herramienta útil para combatir opiniones no fundamentadas (pensamiento no crítico) y acciones irreflexivas. En otras palabras, la posibilidad de establecer una posición crítica protege a los individuos contra la enajenación que tiene lugar cuando una persona $\mathrm{A}$ procura influenciar a una persona $\mathrm{B}$.

De acuerdo a Paul y Elder (2002) al existir información en cantidad referente a cualquier tema es importante que los estudiantes tengan la capacidad de análisis, solo absorbiendo lo relevante de la información, es decir evaluar lo que tiene importancia de lo que no la tiene, lo que involucra destrezas intelectuales que hacen más eficiente el razonamiento y la toma de decisiones de los sujetos frente a las problemáticas que se presente. Asimismo, Zubiría (2010) resalta las competencias para interpretar e inferir como una de las condiciones para lograr un pensamiento crítico; por ello sugiere que se incorporen inferencias inductivas y deductivas al trabajo educativo por lo que urge la necesidad adecuar el currículo de tal forma que este se enfoque en el desarrollo del pensamiento.

Por su lado, Nosich (2003) señala que siempre que razonamos hay alternativas y que estas nos abren caminos nuevos para decidir cuál es el más adecuado. Para Elder (2005) generar soluciones a un problema permite encontrar diferentes maneras de llevar a cabo un mismo trabajo, pero estas serán eficientes en la medida que sean relevantes a la problemática a ello se debe agregar que las propuestas deben plantearse en función del bienestar propio y el de los demás.

Para Boisvert (2004) la opinión o postura sobre un tema debe ser acompañado con la justificación de argumentos. Según Beas, Santa Cruz, Thomsen y Utreras (1995) al elaborar los fundamentos es necesario identificar lo que se conoce del tema, los objetivos que se persiguen, las características del discurso y el público al cual se dirige la argumentación. Beas, Santa Cruz, Thomsen y Utreras (1995) afirma que si los niños aprenden a decir las cosas basándose en argumentos entonces se produce una contribución al desarrollo del pensamiento sensato lo que conlleva a una maduración cognitiva.

De acuerdo a Santrock (2011) sostiene que la preocupación por el pensamiento crítico 
entre maestros y psicólogos no es de interés reciente, el autor hace referencia a John Dewey, filósofo y pedagogo norteamericano, quien hacia 1933 ya hablaba de la importancia de que los estudiantes piensen reflexivamente; y a Max Wertheimer, que en 1945 habló sobre el pensamiento productivo que es contrario al simple hecho de adivinar una respuesta correcta. Para Campos (2007) de acuerdo a un recuento que realiza de la historia del pensamiento crítico usando las edades de la historia universal. Así reconoce en Sócrates y su trascendental mayéutica al más conspicuo representante de la edad antigua. En la edad media señala a Santo Tomás de Aquino como el personaje principal que impulsa el pensamiento crítico, para la edad moderna hace referencia a Thomas Moro, Francis Bacón y René Descartes; mientras que en la edad contemporánea reconoce los aportes de John Dewey, Benjamín Bloom, Richard Paul, Linda Elder, Matthew Lipman, Robert Ennis entre otros.

Al ser el pensamiento crítico una actividad tan compleja, cualquier intento de ofrecer una serie de dimensiones completa y definitiva podría resultar inútil si se aborda desde una única perspectiva. Dentro de la investigación de Milla (2012) se pudo apreciar que existe coincidencia entre los investigadores en señalar dos componentes o dimensiones para el pensamiento crítico, las que involucran aspectos afectivos y aquellas que están relacionadas con aspectos netamente cognitivos.

En los tiempos actuales la información respecto de cualquier tema es abundante, por ello es necesario que los estudiantes pongan en práctica técnicas que los ayuden a separar la información considerando solo aquello que es relevante. Al respecto Raths, Wasserman, Jonas y Rothsein (1999) manifiestan que analizar es discernir y evaluar lo que tiene importancia de lo que no la tiene. Siendo necesario entonces enseñar a los alumnos a distinguir lo significativo de lo no significativo.

Según Figueroa (2004) el rendimiento académico es el conjunto de transformaciones operadas en el educando, a través del proceso enseñanza - aprendizaje, que se manifiesta mediante el crecimiento y enriquecimiento de la personalidad en formación, este mismo autor clasifica el rendimiento académico en dos tipos, individual y social.

\subsection{Metodología}

El estudio es de enfoque cuantitativo, ya que se usó la recolección de datos para probar la hipótesis, con base a la medición numérica y el análisis estadístico, además fue una investigación de alcance explicativo, ya que se centró en el interés de explicar porque 
ocurre un fenómeno y qué condiciones se manifiesta variables o porque se relacionan dos o más variables. Correspondió a una investigación de diseño no experimental, este tipo de investigaciones son estudios que se realizan sin la manipulación deliberada de variables. Asimismo, fue una investigación de diseño transeccional (transversal), la población comprendió a 57 docentes nombrados de la Escuela de Educación los mismos que fueron considerados como parte de la muestra. La técnica empleada para la recopilación de datos fue la encuesta y como instrumento el cuestionario, este instrumento fueron debidamente trabajados para establecer su confiabilidad por el método de Alfa de Cronbach, y su validez se estableció mediante la validación por juicio de expertos. Para el análisis e interpretación de la información se construyó una base de datos, la cual fue exportada al programa estadístico de la IBM SPSS, en su versión 25, en este programa se trabajó los datos, se construyó una matriz de niveles y puntuaciones para alcanzar los objetivos señalados.

\section{RESULTADOS Y DISCUSIÓN}

\subsection{En cuanto al pensamiento crítico y su influencia en el Rendimiento Académico}

De acuerdo a Tabla 1 , se aprecia que del $42,1 \%$ de docentes que perciben que en la escuela de educación existe un nivel bajo en pensamiento crítico, todos también afirman que en la escuela de educación existe un nivel malo en el rendimiento académico. Por el contrario, del 54,4\% de los docentes encuestados que aseguran que en la escuela de educación existe un nivel medio de pensamiento crítico, el 14\% de los docentes perciben que en la escuela de educación existe un nivel malo de rendimiento académico y el 40,4\% de los docentes perciben que la escuela de educación existe un nivel regular en rendimiento académico. Así mismo se encontró que el Pensamiento Crítico influye de manera directa y significativa en el Rendimiento Académico en la Escuela de Educación, de una universidad pública, 2020; esto se aprecia a un coeficiente de correlación de 0,768, evidenciando una correspondencia alta entre el Pensamiento Crítico y el Rendimiento Académico, de igual forma se aprecia un nivel de significancia de 0,000 menor a la significancia teórica $\alpha=0,05$ denotando la influencia de las variables, por lo que se cumple la hipótesis de la investigación. 


\section{Tabla 1}

El Pensamiento Crítico y el Rendimiento Académico en la Escuela de Educación, de una universidad pública, 2020.

\begin{tabular}{|c|c|c|c|c|c|c|}
\hline & & & & $\begin{array}{l}\text { NDIMIEN } \\
\text { ADÉMIC }\end{array}$ & & Total \\
\hline & & & Malo & Regular & Bueno & \\
\hline & & Recuento & 24 & 0 & 0 & 24 \\
\hline & Вајо & $\%$ del total & $42,1 \%$ & $0,0 \%$ & $0,0 \%$ & $42,1 \%$ \\
\hline Pensamiento & & Recuento & 8 & 23 & 0 & 31 \\
\hline crítico & Medio & $\%$ del total & $14,0 \%$ & $40,4 \%$ & $0,0 \%$ & $54,4 \%$ \\
\hline & $\Delta 1 t$ & Recuento & 0 & 1 & 1 & 2 \\
\hline & Allo & $\%$ del total & $0,0 \%$ & $1,8 \%$ & $1,8 \%$ & $3,5 \%$ \\
\hline Tota & & Recuento & 32 & 24 & 1 & 57 \\
\hline I & & $\%$ del total & $56,1 \%$ & $42,1 \%$ & $1,8 \%$ & $100,0 \%$ \\
\hline
\end{tabular}

Fuente: Datos alcanzados en el estudio.

\subsection{En correspondencia al análisis de información y su influencia en el rendimiento académico}

De acuerdo a Tabla 2, se aprecia que del 35,1\% de docentes que perciben que en la escuela de educación existe un nivel bajo de análisis de información, todos también afirman que en la escuela de educación existe un nivel malo en el rendimiento académico. Por el contrario, del 63,2\% de los docentes encuestados que aseguran que en la escuela de educación existe un nivel medio de análisis de información, el 21,1\% de los docentes perciben que en la escuela de educación existe un nivel malo de rendimiento académico, el $40,4 \%$ de los docentes perciben existe un nivel regular de rendimiento académico y solo el 1,8\% de los docentes evidencian que en la escuela de educación existe un nivel bueno en rendimiento académico. Y respecto al primer objetivo específico se encontró que el análisis de información influye favorablemente en el Rendimiento Académico en la Escuela de Educación; esto se aprecia a un coeficiente de correlación de 0,650, evidenciando una correspondencia moderada entre el análisis de información y el Rendimiento Académico, de igual forma se aprecia un nivel de significancia de 0,000 menor a la significancia teórica $\alpha=0,05$, por lo que se cumple la hipótesis de la investigación. 


\section{Tabla 2}

El análisis de información y el Rendimiento Académico en la Escuela de Educación, de una universidad pública, 2020.

\begin{tabular}{|c|c|c|c|c|c|c|}
\hline & & & \multicolumn{3}{|c|}{$\begin{array}{c}\text { RENDIMIENTO } \\
\text { ACADÉMICO }\end{array}$} & \multirow[t]{2}{*}{ Total } \\
\hline & & & Malo & Regular & Bueno & \\
\hline \multirow{6}{*}{$\begin{array}{l}\text { Analizar } \\
\text { información }\end{array}$} & \multirow{2}{*}{ Bajo } & Recuento & 20 & 0 & 0 & 20 \\
\hline & & $\%$ del total & $35,1 \%$ & $0,0 \%$ & $0,0 \%$ & $35,1 \%$ \\
\hline & \multirow{2}{*}{ Medio } & Recuento & 12 & 23 & 1 & 36 \\
\hline & & $\%$ del total & $21,1 \%$ & $40,4 \%$ & $1,8 \%$ & $63,2 \%$ \\
\hline & \multirow{2}{*}{ Alto } & Recuento & 0 & 1 & 0 & 1 \\
\hline & & $\%$ del total & $0,0 \%$ & $1,8 \%$ & $0,0 \%$ & $1,8 \%$ \\
\hline \multirow{2}{*}{ Total } & & Recuento & 32 & 24 & 1 & 57 \\
\hline & & $\%$ del total & $56,1 \%$ & $42,1 \%$ & $1,8 \%$ & $100,0 \%$ \\
\hline
\end{tabular}

Fuente: Datos alcanzados en el estudio.

\subsection{En relación a la inferencia de implicancias y su influencia en el rendimiento académico}

De acuerdo a Tabla 3, se aprecia que del 52,6\% de docentes que perciben que en la escuela de educación existe un nivel bajo en inferencia de implicancias, el 49,1\% de docentes afirman que en la escuela de educación existe un nivel malo de rendimiento académico y solo el 3,5\% afirman que existe un nivel regular de rendimiento académico. Por el contrario, del 45,6\% de los docentes encuestados que aseguran que en la escuela de educación existe un nivel medio en inferencia de implicancias, solo el $7 \%$ de los docentes perciben un nivel malo de rendimiento académico y el 38,6\% de los docentes perciben existe un nivel regular de rendimiento académico. Del mismo modo, se encontró que la inferencia de implicancias influye positiva y significativamente en el Rendimiento Académico en la Escuela de Educación; esto se aprecia a un coeficiente de correlación de 0,800 , evidenciando una correspondencia alta entre la inferencia de implicancias y el Rendimiento Académico, de igual forma se aprecia un nivel de significancia de 0,000 menor a la significancia teórica $\alpha=0,05$, por lo que se cumple la hipótesis de la investigación. 


\section{Tabla 3}

La inferencia de implicancias y el Rendimiento Académico en la Escuela de Educación, de una universidad pública, 2020.

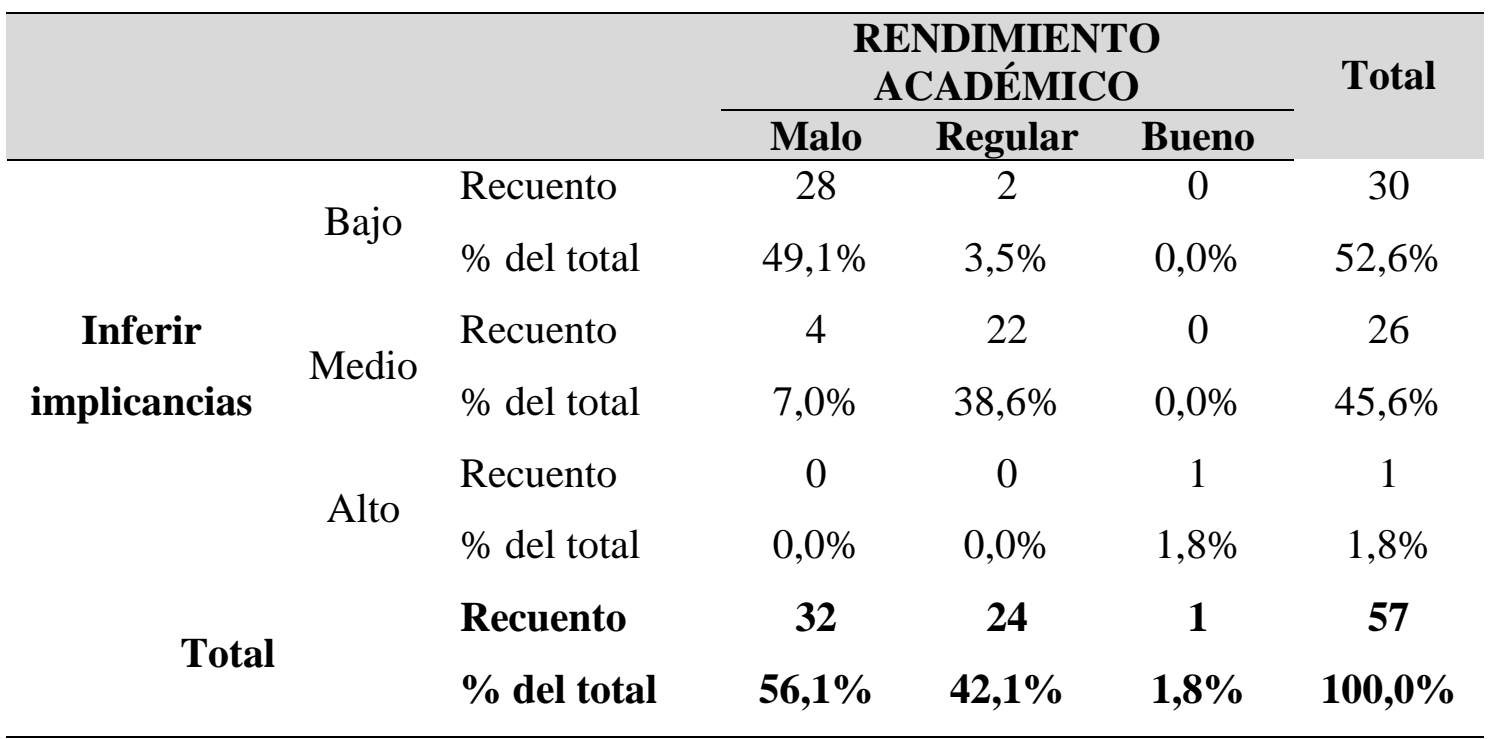

Fuente: Datos alcanzados en el estudio.

\subsection{En referencia a como el proponer alternativas de solución influye en el} Rendimiento Académico

De acuerdo a Tabla 4, se aprecia que del 31,6\% de docentes que perciben que en la escuela de educación existe un nivel bajo de propuestas de alternativas de solución, todos también afirman que existe un nivel malo en el rendimiento académico. Por el contrario, del 66,7\% de los docentes encuestados que aseguran que en la escuela de educación existe un nivel medio de propuestas de alternativas de solución, el 24,6\% perciben que existe un nivel malo de rendimiento académico, el 40,4\% perciben que existe un nivel regular de rendimiento académico y solo el 1,8\% evidencian que existe un nivel bueno en rendimiento académico. También se encontró que la propuesta de alternativas de solución influye positiva y significativamente en el Rendimiento Académico en la Escuela de Educación, de una universidad pública, 2020; esto se aprecia a un coeficiente de correlación de 0,603, evidenciando una correspondencia moderada entre la propuesta de alternativas de solución y el Rendimiento Académico, de igual forma se aprecia un nivel de significancia de 0,000 menor a la significancia teórica $\alpha=0,05$, por lo que se cumple la hipótesis de la investigación. 


\section{Tabla 4}

La propuesta de alternativas de solución y el Rendimiento Académico en la Escuela de

Educación, de una universidad pública, 2020.

\begin{tabular}{|c|c|c|c|c|c|c|}
\hline & & & \multicolumn{3}{|c|}{$\begin{array}{c}\text { RENDIMIENTO } \\
\text { ACADÉMICO }\end{array}$} & \multirow[t]{2}{*}{ Total } \\
\hline & & & Malo & Regular & Bueno & \\
\hline \multirow{6}{*}{$\begin{array}{c}\text { Proponer } \\
\text { alternativas de } \\
\text { solución }\end{array}$} & \multirow{2}{*}{ Bajo } & Recuento & 18 & 0 & 0 & 18 \\
\hline & & $\%$ del total & $31,6 \%$ & $0,0 \%$ & $0,0 \%$ & $31,6 \%$ \\
\hline & \multirow{2}{*}{ Medio } & Recuento & 14 & 23 & 1 & 38 \\
\hline & & $\%$ del total & $24,6 \%$ & $40,4 \%$ & $1,8 \%$ & $66,7 \%$ \\
\hline & \multirow{2}{*}{ Alto } & Recuento & 0 & 1 & 0 & 1 \\
\hline & & $\%$ del total & $0,0 \%$ & $1,8 \%$ & $0,0 \%$ & $1,8 \%$ \\
\hline \multirow{2}{*}{ Total } & & Recuento & 32 & 24 & 1 & 57 \\
\hline & & $\%$ del total & $56,1 \%$ & $42,1 \%$ & $1,8 \%$ & $100,0 \%$ \\
\hline
\end{tabular}

Fuente: Datos alcanzados en el estudio.

\subsection{En correspondencia al argumento y su influencia en el Rendimiento Académico en la Escuela}

De acuerdo a Tabla 5, se aprecia que del 38,6\% de docentes que perciben que en la escuela de educación existe un nivel bajo de argumentación, todos también afirman que en la escuela de educación existe un nivel malo en el rendimiento académico. Por el contrario, del 57,9\% de los docentes encuestados que aseguran que en la escuela de educación existe un nivel medio de argumentación, el 17,5\% de los docentes perciben que en la escuela de educación existe un nivel malo de rendimiento académico y el 40,4\% de los docentes perciben que la escuela de educación existe un nivel regular en rendimiento académico. Y del 3,5\% de los docentes encuestados que aseguran que existe un nivel alto de argumentación en la escuela de educación, el 1,8\% percibe que en la escuela de educación existe un nivel regular en rendimiento académico y el otro $1,8 \%$ de docentes afirman que hay un nivel bueno en rendimiento académico. Asi también se encontró que la argumentación influye de manera positiva y significativa en el Rendimiento Académico en la Escuela de Educación; esto se aprecia a un coeficiente de correlación de 0,603, evidenciando una correspondencia moderada entre la argumentación y el Rendimiento Académico, de igual forma se aprecia un nivel de significancia de 0,000 menor a la significancia teórica $\alpha=0,05$, por lo que se cumple la hipótesis de la investigación. 


\section{Tabla 5}

La argumentación y el Rendimiento Académico en la Escuela de Educación, de una universidad pública, 2020.

\begin{tabular}{|c|c|c|c|c|c|c|}
\hline & & & & $\begin{array}{l}\text { NDIMIEN } \\
\text { ADÉMIC }\end{array}$ & & \\
\hline & & & Malo & Regular & Bueno & Total \\
\hline & & Recuento & 22 & 0 & 0 & 22 \\
\hline & Вајо & $\%$ del total & $38,6 \%$ & $0,0 \%$ & $0,0 \%$ & $38,6 \%$ \\
\hline Argumentar & Modit & Recuento & 10 & 23 & 0 & 33 \\
\hline posición & IVIedio & $\%$ del total & $17,5 \%$ & $40,4 \%$ & $0,0 \%$ & $57,9 \%$ \\
\hline & Alto & Recuento & 0 & 1 & 1 & 2 \\
\hline & กाषण & $\%$ del total & $0,0 \%$ & $1,8 \%$ & $1,8 \%$ & $3,5 \%$ \\
\hline Total & & Recuento & 32 & 24 & 1 & 57 \\
\hline Total & & $\%$ del total & $56,1 \%$ & $42,1 \%$ & $1,8 \%$ & $100,0 \%$ \\
\hline
\end{tabular}

Fuente: Datos alcanzados en el estudio.

\subsection{Discusión}

Respecto a la hipótesis general, se encontró que el Pensamiento Crítico influye de manera directa y significativa en el Rendimiento Académico en la Escuela de Educación, de una universidad pública, 2020; esto se aprecia a un coeficiente de correlación de 0,768, evidenciando una correspondencia alta entre el Pensamiento Crítico y el Rendimiento Académico, de igual forma se aprecia un nivel de significancia de 0,000 menor a la significancia teórica $\alpha=0,05$ denotando la influencia de las variables, por lo que se cumple la hipótesis de la investigación.

Este resultado es similar a lo encontrado por Huaranca (2018) de acuerdo con el desarrollo de su investigación se observó que el existe una relación significativa entre el pensamiento crítico y el rendimiento académico, siendo que la prueba de correlación resulto con un coeficiente de 0.643 , concluyendo que el pensamiento crítico influye de forma moderada y directa al rendimiento crítico. Del Busto (2017) que de acuerdo con su investigación el pensamiento crítico se relaciona de forma positiva con el rendimiento académico, siendo esta correlación significativa, positiva y moderada de 0.81 demostrando la correlación de las variables. 
Se contrasta con los resultados obtenidos por Alquichire y Arrieta (2018) en la investigación sobre el pensamiento crítico y el rendimiento académico, donde se observó que las variables en estudio presentan una correlación negativa, al presentarse en la prueba de correlación de $0.004<0,05$, ello mediante el estudio de una muestra de estudiantes de primer semestre de la Licenciatura en Biología y Química de la Universidad del Atlántico. El pensamiento crítico se refleja como la búsqueda crítica y reflexiva de conclusiones válidas que resuelvan problemas y dudas, permitiendo elegir entre afirmaciones antagónicas (Burton, Kimball, \& Wing, 1965)(Burton et al., 1965), a medida que el pensamiento se mejora se va dando lugar a la transformación del mismo en pensamiento crítico, ya que al ser un proceso consciente que se utiliza para realizar interpretaciones de información fundamentada y basada en conocimientos que conduce al juicio porque apoya los criterios siendo sensible al contexto en que se maneje. Lipman (1991) procesos críticos de pensamiento que les permiten distinguir la información más relevante de la menos relevante en relación con sus metas, siendo una herramienta útil que protege a los individuos contra la enajenación que tiene lugar cuando una persona procura influenciar a otra persona. Según los resultados obtenidos en la investigación se observó que el pensamiento crítico influye en el rendimiento académicos de los estudiantes.

Respecto a la primera hipótesis específica, se encontró que el análisis de información influye favorablemente en el Rendimiento Académico en la Escuela de Educación; esto se aprecia a un coeficiente de correlación de 0,650, evidenciando una correspondencia moderada entre el análisis de información y el Rendimiento Académico, de igual forma se aprecia un nivel de significancia de 0,000 menor a la significancia teórica $\alpha=0,05$, por lo que se cumple la hipótesis de la investigación.

Este resultado es similar a lo encontrado por Del Bustos (2017) donde señala que existe correlación directa y significativa fuerte entre el análisis de información y el rendimiento académico. Macedo (2018) donde señala que, el análisis de información se relaciona con el rendimiento académico, esto a una distribución normal con un nivel de significancia mayor al 5\% en este caso $19.9 \%(\mathrm{P}=.199>.05)$.

Se contrasta con los resultados obtenidos por Alquichire y Arrieta (2018) en su investigación donde se observa que entre el análisis y el rendimiento presentaron un 
coeficiente de correlación con una significancia de 0.0012 , por lo que no una correlación entre el análisis y el rendimiento académico.

Al existir información en cantidad referente a cualquier tema es importante que los estudiantes tengan la capacidad de análisis, solo absorbiendo lo relevante de la información, es decir evaluar lo que tiene importancia de lo que no la tiene, lo que involucra destrezas intelectuales que hacen más eficiente el razonamiento y la toma de decisiones de los sujetos frente a las problemáticas que se presente (Paul y Elder, 2003). Según los resultados obtenidos en la investigación el realizar un buen análisis de información ayuda a un mejor rendimiento académico de los estudiantes.

Respecto a la segunda hipótesis específica, es similar a la investigación realizada por Del Bustos (2017) donde señala que existe relación entre el inferir implicancias y el rendimiento académico. Contrasta con los resultados obtenidos por Alquichire y Arrieta (2018) donde se concluye, aunque los estudiantes se ubicaron en la categoría Promedio de la habilidad de inferencia evaluadas, esto no significó un muy buen rendimiento académico en términos generales, por lo se obtuvo que las inferencias no influyen de forma significativa en el rendimiento académico.

Inferir implicancias es una destreza de realizar predicciones razonables sobre los efectos de una situación a partir de datos explícitos, por tanto, es resultado de la deducción y la activación de saberes previos factores que al interactuar dan lugar a la producción de conclusiones. Zubiría (2010) recalca que interpretar es una de las condiciones importantes para que el sujeto desarrolle el pensamiento crítico y al ser desarrollado la mente llega resolver alguna problemática. Por lo que según los resultados obtenidos en la investigación observo que el inferir implicancias influye el rendimiento académico de los estudiantes.

Respecto a la tercera hipótesis específica, se encontró que la propuesta de alternativas de solución influye positiva y significativamente en el Rendimiento Académico en la Escuela de Educación, de una universidad pública, 2020; esto se aprecia a un coeficiente de correlación de 0,603, evidenciando una correspondencia moderada entre la propuesta de alternativas de solución y el Rendimiento Académico, de igual forma se aprecia un nivel de significancia de 0,000 menor a la significancia teórica $\alpha=0,05$, por lo que se cumple la hipótesis de la investigación. 
Es similar a la investigación realizada por Huaranca (2018) donde señala que existe influencia entre alternativas múltiples de solución y el rendimiento, con un coeficiente de correlación de 0.543. La capacidad que los estudiantes tengan para establecer diferentes y posibles respuestas a los problemas que analizan ayuda a forma un pensamiento crítico que se va a ir moldeando realizando ejercicios seguidos de empatía cognitiva puesto que las soluciones irán de acuerdo a la realidad en que se coloquen. Para lo Nosich (2003) señala que siempre que razonamos hay alternativas y que estas nos abren caminos nuevos para decidir cuál es mejor. Por lo que según los resultados obtenidos se observó que el plantear alternativas de solución ayudara a mejorar el rendimiento académico de los estudiantes, el producir alternativas de solución hace que la participación personal se vaya fortaleciendo en resolución de conflictos que lleguen a presentarse.

Respecto a la cuarta hipótesis específica, se encontró que la argumentación influye de manera positiva y significativa en el Rendimiento Académico en la Escuela de Educación; esto se aprecia a un coeficiente de correlación de 0,603, evidenciando una correspondencia moderada entre la argumentación y el Rendimiento Académico, de igual forma se aprecia un nivel de significancia de 0,000 menor a la significancia teórica $\alpha=$ 0,05 , por lo que se cumple la hipótesis de la investigación.

Es similar a la investigación realizada por Huaranca (2018) donde señala que existe influencia entre la argumentación y el rendimiento, con un coeficiente de método correlación de 0.593 , mostrando una correlación moderada. La argumentación es la mejor herramienta que permite a los estudiantes a demostrar las evidencia que van a servir de apoyo a los planteamientos que hagan sobre un tema, ya que al ser afirmaciones que tienen respaldo en base a un teoría o investigación demostraran la veracidad de sus ideas (Beas et al. 1995). Por lo que, un estudiante que tiene una correcta argumentación de sus ideas y pesamientos va a obtener un mejor redimiento academico según los resultados que se han obtenidos en la investigación, una persona al dar su posición basado en un argumento contribuye al desarrollo del pensamiento crítico llevando a una maduracion cognitiva, reflejandose en el rendimiento académico.

Estos resultados han permitido aceptar la hipotesis propuesta de la investigación, por lo que existe influencia del pensamiento critico en el rendimeinto académico, desde esta perpetiva es importante que los estudiantes desarrollen de forma adecuada el pensamiento 
haciendolos muchos mas competentes y eficientes en cualquier actividad que deseen realizar

\section{CONSIDERACIONES FINALES}

Se llega a concluir que el Pensamiento Crítico influye de manera directa y significativa en el Rendimiento Académico en la Escuela de Educación; esto se aprecia a un coeficiente de correlación Rho de Spearman de 0,768, evidenciando una correspondencia alta entre el Pensamiento Crítico y el Rendimiento Académico, de igual forma se aprecia un nivel de significancia de 0,000 menor a la significancia teórica $\alpha=0.05$ denotando un relación significativa, por lo que se cumple la hipótesis de investigación.

Se concluye también que el análisis de información influye favorablemente en el Rendimiento Académico en la Escuela de Educación; esto se aprecia a un coeficiente de correlación Rho de Spearman de 0,650, evidenciando una correspondencia moderada entre el análisis de información y el Rendimiento Académico de igual forma se aprecia un nivel de significancia de 0,000 menor a la significancia teórica $\alpha=0.05$ denotando una relación significativa, por lo que se cumple la hipótesis de investigación.

Se comprobó que la inferencia de implicancias influye positiva y significativamente en el Rendimiento Académico en la Escuela de Educación; esto se aprecia a un coeficiente de correlación Rho de Spearman de 0,800, evidenciando una correspondencia alta entre la inferencia de implicancias y el Rendimiento Académico de igual forma se aprecia un nivel de significancia de 0,000 menor a la significancia teórica $\alpha=0.05$ denotando una relación significativa, por lo que se cumple la hipótesis de investigación.

Se encontró que la propuesta de alternativas de solución influye positiva y significativamente en el Rendimiento Académico en la Escuela de Educación; esto se aprecia a un coeficiente de correlación Rho de Spearman de 0,603, evidenciando una correspondencia moderada entre la propuesta de alternativas de solución y el Rendimiento Académico de igual forma se aprecia un nivel de significancia de 0,000 menor a la significancia teórica $\alpha=0.05$ denotando un relación significativa, por lo que se cumple la hipótesis de investigación.

Se concluye que la argumentación influye de manera positiva y significativa en el Rendimiento Académico en la Escuela de Educación; esto se aprecia a un coeficiente de correlación Rho de Spearman de 0,603, evidenciando una correspondencia moderada entre la argumentación y el Rendimiento Académico, de igual forma se aprecia un nivel 
de significancia de 0,000 menor a la significancia teórica $\alpha=0,05$, por lo que se cumple la hipótesis de la investigación de igual forma se aprecia un nivel de significancia de 0,000 menor a la significancia teórica $\alpha=0.05$ denotando un relación significativa, por lo que se cumple la hipótesis de investigación.

\section{LISTA DE REFERENCIAS (IDEAL AL MENOS 20 FUENTES CITADAS)}

Alquichire, S., \& Arrieta, J. (2018). Relación entre habilidades de pensamiento crítico y rendimiento académico. Barranquilla: Universidad del Atlántico.

Beas, J., Santa Cruz, J., Thomsen, P., \& Utreras, S. (1995). Enseñar a pensar para aprender mejor. Santiago: Universidad Católica de Chile.

Boisvert, J. (2004). La formación del pensamiento crítico. México D.F: Fondo de Cultura Económica.

Burton, W., Kimball, R., \& Wing, R. (1965). Hacia un pensamiento eficaz. Buenos Aires: Troquel.

Campos, A. (2007). Pensamiento Crítico. Técnicas para su Desarrollo. Bogotá: Magisterio.

Del Busto, R. (2017). Pensamiento Crítico y Rendimiento Académico en el área de Formación Ciudadana y Cívica en alumnos de cuarto y quinto de secundaria de una institución Educativa Estatal del Distrito de Los Olivos. Lima: Universidad Ricardo Palma.

Del Busto, R. (2017). Pensamiento Crítico y Rendimiento Académico en el área de Formación Ciudadana y Cívica en alumnos de Cuarto y Quinto de Secundaria de una Institución Educativa Estatal del Distrito de Los Olivos (Tesis de posgrado). Lima: Universidad Ricardo Palma. Obtenido de http://repositorio.urp.edu.pe/bitstream/handle/URP/1577/REDELBUSTOB.pdf? sequence $=1 \&$ isAllowed $=\mathrm{y}$

Figueroa, C. (2004). Sistemas de evaluación académica. El Salvador: Universitaria.

Huaranca, I. (2018). Relación entre el pensamiento crítico y el rendimiento académico en estudiantes de la escuela profesional de Contabilidad de la Universidad Católica los Ángeles de Chimbote filial Juliaca año 2018. Chimbote: Universidad Católica los Ángeles de Chimbote.

Lipman, M. (1991). Thinking in education. Cambridge: Cambridge University Press. 
Macedo, A. (2018). pensamiento crítico y rendimiento académico en los ingresantes del curso de Estadística I en la Facultad de Ingeniería Económica, Estadística y Ciencias Sociales. Universidad Nacional de Ingeniería - 2018 (Tesis de psgrado). Lima: Universida Calletano Heredia. Obtenido de http://repositorio.upch.edu.pe/bitstream/handle/upch/1541/Pensamiento_Macedo Davila_Antonieta.pdf?sequence $=1 \&$ isAllowed $=\mathrm{y}$

Mertes, L. (1991). Thinking and Writing. Middle School Journal, 24-25.

Milla, M. (2012). Pensamiento crítico en estudiantes de quinto de secundaria de los colegios de Carmen de la Legua, Callao. Lima: Universidad San Ignacio de Loyola.

Nosich, G. (2003). Aprender a pensar, pensamiento analítico para estudiantes. Madrid: Pearson Education.

Paul, R., \& Elder, L. (2002). Critical Thinking: Tools for Taking Charge of Your Professional \& Personal Life. Nueva Jersey: Prentice Hall.

Raths, Y., Wasserman, S., Jonas, A., \& Rothsein, A. (1999). Como enseñar a pensar. México D.F: Paidós.

Santrock, J. (2011). Psicologia de la Educacion 4ta Edición. España: S.A. MCGRAWHILL / Interamericana de España.

Zubiría, J. (2010). Estrategias para el desarrollo del pensamiento crítico. Lima: Instituto de Pedagogía Popular. 